\title{
HGM2008 infectious diseases and metagenomics symposium abstracts
}

\section{1: M. tb. PE/PPE family genes: Role in Quorom sensing and modulating the host response}

\section{Seyed E. Hasnain \\ Center for DNA Fingerprinting and Diagnostics (CDFD), Hyderabad 500 076, India}

Death due to TB caused by the pathogen Mycobacterium tuberculosis (M. $t b$.) accounts approximately for one death every $15 \mathrm{~s}$ somewhere in the world. The most interesting outcome of the genome sequence data of $M$. $t b$. was the presence of a large number of hypothetical genes or those for which functions could only be ascribed based on homology and also the presence of a small number (about 10\%) of genes belonging to the PE/PPE family which have no known homologues in the living kingdom other than in the genus Mycobacteria. Molecular epidemiology, evolutionary dynamics and the functional biology of $M$. $t b$. has been the major interest of my group. We are focusing on genes which are unknown, and also those known to have a function, with a view to understand how $M$. $t b$. has evolved unique modalities to evade the immune system. In the process we have additionally been able to identify new diagnostic antigens and at the same time potential drug targets. Our studies have also shown that immunology crosstalk's between host (human) and the pathogen $(M . t b$.) involve the use of select members of the large PE and PPE proteins family as well as other essential enzymes known to have non-immune function. My presentation will focus on the host parasite interactions aimed at crippling the host immune response which the pathogen $(M . t b$.) very cleverly initiates for its own survival and dissemination.

\section{3: Metagenomics of the human gut microbiome}

\section{Todd D. Taylor}

MetaSystems Research Team, Computational Systems Biology Research Group, Advanced Computational Sciences Department, RIKEN Advanced Science Institute, 1-7-22 Suehiro-cho, Tsurumi-ku, Yokohama, Kanagawa 230-0045, Japan

An enormous number of microorganisms, the vast majority of which are bacteria, colonize and form specific communities (microbiota) at many human body sites. The human microbiota, including the most abundant gut microbiota, together with host cells forms a complex ecosystem that collaboratively conducts various biological processes. Thus, we must consider ourselves as 'super organisms' made up of both human cells and our indigenous microbes, whose composite genome is referred to as the human 'metagenome'. The average human adult gut microbiota comprises $10^{9-11}$ out of an estimated $10^{14}$ total commensal microbes per individual, accounting for approximately 10 times the number of eukaryotic cells in the human body. These gut microbiota are largely associated with the metabolism processes necessary in human biology, maturation and proliferation of host intestinal cells to maintain gut homeostasis, and etiology of various diseases such as inflammatory bowel diseases and obesity. The International Human Microbiome Consortium (IHMC), which was launched in 2007, aims to collect and integrate the genomic information of several medically important human microbiomes. To explore the genomic and functional features of complex microbial communities, including those of uncultivable microbes, a culture-independent metagenomic approach is necessary. Our group (The Human Metagenome Consortium Japan; HMGJ) has analyzed the gut microbiomes of 13 healthy Japanese individuals, including adults, weaned children, and unweaned infants. We generated about 1 million Sanger reads accounting for unique metagenomic sequences totalling $479 \mathrm{Mb}$ and predicted about 660,000 bacterial genes in them. By searching for the genes significantly enriched in gut microbiomes in contrast with those present in non-gut microbiomes, we identified 315 enriched gene families in these 13 human microbiomes. Analysis of their predicted functions indicated the abundance of functions involved in carbohydrate metabolism as well as many conserved gene families of unknown function, suggesting that these gene sets may encode the core functions necessary to adapt to the gut habitat. We also identified 647 novel gene families specific to human gut microbiomes by comparing with genes present in the metagenomic data of other microbial communities such as sea-surface, deep-sea and soil. The IHMC, including the activities of HMGJ, will further promote this research field and pave the way to a new frontier of human biology which is expected to develop new strategies for actively sustaining human health.

\section{4: From bench to bedside to populations: Translation of CCL3L1-CCR5 genetics for prevention and management of HIV-AIDS}

\section{Sunil K. Ahuja}

University of Texas Health Science Center at San Antonio, 7703 Floyd Curl Dr, San Antonio, TX 78229, United States of America 
Borrowing from Shakespeare, (William Shakespeare, and King Richard II. Act ii. Sc. 1) "...this other Eden, demi-paradise, this fortress built by Nature for herself against infection..."

Nature's fortress against infections such as HIV by necessity involves host factors that influence the early events of HIV-host interactions. In this seminar, I will show how we have used a genetic approach as a powerful tool to identify the host factors that underlie some of these early events. This approach has therefore permitted us to address the following clinical paradoxes: Why do some individuals when exposed to HIV-1 resist infection? Why do some individuals succumb to HIV-1 infection rapidly whereas others resist progression to AIDS? Why do some individuals have a better recovery of immune function after initiation of HAART?

Using this genetic approach we have (a) uncovered complex host gene-gene interactions that influence HIV-1 pathogenesis in vivo; (b) determined the relative contribution of some of these host genetic determinants to the HIV-1 epidemic at the population level. Specifically, in this seminar, I will focus on the role of CCR5 and its ligands in HIV/AIDS pathogenesis. In previous studies we uncovered some of the the CCR5 genetic determinants that contribute to inter-subject differences in HIV/AIDS susceptibility. Here, I will show that there are significant interindividual and interpopulation differences in the copy number of a segmental duplication encompassing the gene encoding CCL3L1 (MIP- $1 \alpha \mathrm{P}$ ), a potent HIV-1-suppressive chemokine and ligand for the HIV coreceptor CCR5. Possession of a CCL3L1 copy number lower than the population average is associated with markedly enhanced HIV/AIDS susceptibility. This susceptibility is even greater in individuals who also possess diseaseaccelerating CCR5 genotypes. This relationship between CCL3L1 dose and altered HIV/AIDS susceptibility points to a central role for CCL3L1 in HIV/AIDS pathogenesis. I will show that these genetic factors influence HIV pathogenesis by affecting both viral entrydependent and -independent processes, one of which we identify as cell-mediated immunity. I will then place these findings in the broader context of issues relevant to the epidemic/vaccines as well as individual-specific issues such as whether these variants have relevance for understanding the inter-subject differences in response to antiretroviral therapy. 